\title{
RELACIÓN DE AYUDA EN ENFERMERÍA, VISIÓN ANTROPOLÓGICA DE LA COMPETENCIA RELACIONAL EN EL PREGADO ESEN/FACS/UNJBG - 2010
}

\author{
HOW-TO RELATION AT INFIRMARY, ANTHROPOLOGICAL VISION OFTHE \\ COMPETITION RELACIONAL IN THE PRE-DEGREE ESEN/FACS/UNJBG - \\ 2010
}

\author{
Jenny C. Mendoza Rosado
}

RESUMEN

\begin{abstract}
La presente investigación cualitativa (fenomenológica) nos permite auto-examinar el quehacer del Enfermero / a en el desarrollo de la competencia relacional a lo largo de su formación académica. La Relación de ayuda es un recurso terapéutico (Psicología) que brinda al personal de salud la oportunidad de contribuir al bienestar de la persona, mejorando los procesos de interrelación. El personal de enfermería quien cuida ininterrumpidamente de ellos, requiere competencias y recursos necesarios para establecer la relación enfermero - paciente. Uno de los objetivos fue Identificar las habilidades y actitudes que desarrollan los estudiantes de pre grado en procesos de Relación de ayuda a pacientes. La muestra de estudio estuvo constituida por 22 estudiantes de pregrado de Enfermería de 2do a 5 to año, quienes realizan práctica comunitaria y hospitalaria. Se utilizaron 2 entrevistas estructuradas en categorías y subcategorias, aplicadas en forma presencial. Se concluye que la Relación de ayuda que brinda el estudiante de pregrado de Enfermería es limitada (mínimo-positiva), guardando una relación directa entre enseñanza y aplicación práctica, manifestado por la no especificidad de la enseñanza en Relación de ayuda lo que no le permite al estudiante el desarrollo de su competencia relacional.
\end{abstract}

Palabras clave: Relación de ayuda, competencia relacional, visión antropológica, pregrado

\section{ABSTRACT}

\begin{abstract}
This reserch is a qualitative investigation (Phenomenológical), allows us to examine nurse performance on the task of developing interpersonal competency throught his academic formation. The help Relationship is a therapeutic resource (Psychology) that offers to the staff of health the opportunity to contribute to the well-being of the person improving the processes of interrelation. The nursing staff who takes care of them uninterruptedly require competencies and necessary resources to establish the relation nurse - patient. One aim the study was to identify the skills and behaviors that pre-degree of 2 do to 5 to year student develop, who accomplish communal and hospitable practice. Used 2 interviews structured in categories and subcategories were used, applied in eyewitness form. It is concluded that the help relationship that the student of nursing predegree offers is limited (positive minimum), saving a direct account between teaching and practical application, manifested for the low specificity of the teaching in help relationship which does not allow the development of the student relational competency.
\end{abstract}

Keywords: How-to relation, competition relacional, anthropological vision, pre-degree

\section{INTRODUCCIÓN}

La ciencia de Enfermería considera en muchos de sus marcos teóricos lo humanístico y existencial, interesándose cada vez más en conocer al hombre a través de sus comportamientos, experiencia humana y los significados que las cosas tienen para él y cómo experimenta en sí mismo su existencia.

En educación la competitividad y acreditación de la calidad a nivel internacional y nacional ha exigido que todos y entre ellos la profesión de Enfermería avance acorde a dichos procesos y ha considerado en su currículo un enfoque antropológico y transcultural, ya que toda situación de "cuidado de enfermería" en sí es una situación antropológica, pues toda persona enfrenta y responde de diversa manera ante la enfermedad. La Enfermería aporta a la Antropología la visión integral del ser humano a través del "cuidar" a la persona enferma [1]. El cuidado de enfermería como competencia, requiere saberes cognitivos, procedimentales y 
actitudinales para articular la teoría con la práctica, aspectos involucrados en el proceso enseñanza aprendizaje en el pre grado.

La Relación de ayuda o relación terapéutica es un recurso usado continuamente por el estudiante de pregrado de Enfermería en su trato con la persona que recibe los cuidados; por lo que previamente debe haber recibido enseñanza en cuanto a desarrollo de actitudes y habilidades como la empatía, aceptación, escucha activa, confrontar, identificar necesidades espirituales, manejo de tiempo, comunicación no verbal y contacto con la propia experiencia." [2]

Las Teorías de Enfermería como las formuladas por Leininger, Peplau, Henderson, Watson, entre algunas de las que enfatizan la Relación de Ayuda, nos brindan aportes para enseñary aplicar la misma.

Se ha observado que en el plan de estudios de la Facultad de Enfermería/UNJBG, las asignaturas donde se enseña de manera indirecta la Relación de ayuda están ubicadas sólo entre el ler a 3er año, en 1ro. 4 asignaturas, en 2 do. 2 , y en 3ro. 1 asignatura. En la discusión prevalece un sentimiento generalizado de no conocer ni haber recibido enseñanza adecuada del proceso de Relación de Ayuda durante el acompañamiento a los pacientes y familia; en cuanto a Actitudes y Habilidades a nivel individual y grupal manifiestan no haber recibido la enseñanza y motivación para mejorar su competencia relacional en la relación de ayuda, referente a Empatía tienen el conocimiento literal del término, más en la práctica les es difícil diferenciar su aplicación, la aceptación positiva algunas veces no es bien tolerada por el estudiante debido al estado de salud, higiene o gravedad del paciente y otras por la irresponsabilidad que la familia tiene del cuidado de su salud; la escucha activa no fue factible practicarlo pues les ocasiona llamadas de atención por parte de jefes de práctica y otras enfermeras de los servicios de salud, pues lo consideran entretenimiento o pérdida de tiempo; en la confrontación del acompañamiento de situaciones de salud donde el estudiante brinda educación al paciente y familia, los estudiantes refieren que pocas veces el paciente o familia solicitan más información de la otorgada pues generalmente les entienden, participan y colaboran en los procedimientos o intervenciones de Enfermería; las necesidades espirituales casi siempre son aliviadas con un toque en el hombro, conectándolo con la familia y orando con ellos; el manejo del tiempo está muy limitado a la parte práctica y muy superficial en lo que a relaciones interpersonales se refiere, los estudiantes manifiestan que están más interesados en completar la experiencia procedimental, pero si se establecen diálogos prolongados entre los miembros del equipo de salud y es mínimo el contacto con el paciente y familia; la comunicación no verbal es más significativa en la relación estudiante - paciente y familia, quienes manifiestan que entre las expresiones más gratificantes para el paciente está el cogerle la mano, brindarle una mirada, y hacer un gesto de comprensión; finalmente el contacto con la propia experiencia le permite al estudiante brindar cuidados de enfermería comprendiendo el mundo interior del paciente y familia, ya que al haberle tocado vivir una situación similar a la del ser cuidado le permite hablarle desde la experiencia misma, es decir de humano a humano.

\section{OBJETIVOS:}

Identificar las habilidades y actitudes que desarrollan los estudiantes de pre grado en procesos de Relación de ayuda a pacientes.

Establecer si la relación de ayuda en su competencia relacional con el paciente, guarda relación con el nivel de enseñanza en pre grado.

\section{II.-MATERIAL Y MÉTODOS}

\section{1.-Descripción}

La investigación es de tipo cualitativa fenomenológica. Tiene como propósito analizar el proceso de formación en Relación de Ayuda en aspectos teórico práctico, inicial y permanente en los estudiantes de enfermería, que cursan estudios en el año 2010.

\section{2.-Metodología}

Se tomó una muestra representativa de la población estudiantil de la Facultad de Enfermería /UNJBG, constituida por 22 estudiantes de 2 do. a 5 to año de estudios. Participaron sólo estudiantes que asistían en forma regular, 7 de 2 do., 3 de 3 ro, 5 de 4 to. y 7 de 5 to. año; quienes realizan prácticas pre profesionales en servicios hospitalarios y comunitarios de la ciudad de Tacna.

La investigación comprendió 3 fases, recolección de datos a través de entrevista estructurada presencial individual y grupal. En la segunda fase se analizaron las respuestas obtenidas, y en la tercera se continuo con la descripción e interpretación de los datos recogidos. Se aplicaron 2 entrevistas estructuradas con preguntas, 23 abiertas y 9 
cerradas. Para su interpretación se utilizaron 5 niveles referidos a estrategias y habilidades en la relación de ayuda, dificultades y experiencia educativa en el pre grado. Las consideraciones éticas de consentimiento informado para su participación, también fueron incluidas.

\section{III.- RESULTADOS}

Para el análisis de aspectos relevantes de la Investigación, se presentan las categorías y subcategorías tomadas en cuenta, algunos de los cuales se detallarán respectivamente por la naturaleza de la misma.

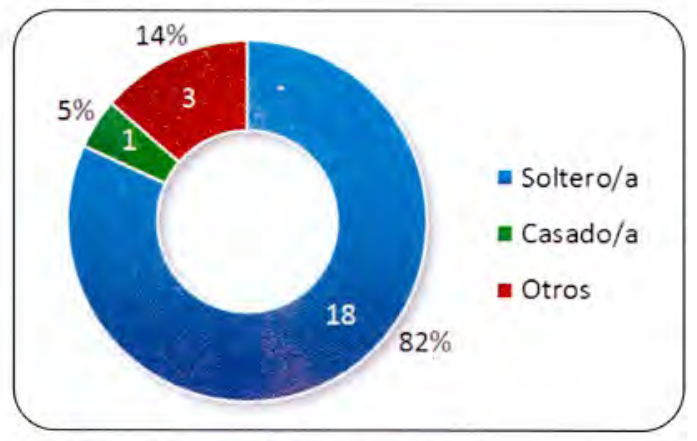

Gráfico $\mathbf{N}^{\circ}$ o1. Distribución de estudiantes según estado civil.
En el Gráfico $\mathrm{N}^{\circ} 01$ se muestra que el $82 \%$ (representados por 18 estudiantes de enfermería) son solteros, el $14 \%$ (3) son casados y convivientes, y el $5 \%$ (1) corresponde a madre soltera.

Tabla $\mathbf{N}^{\circ} \mathbf{0 1}$. Distribución de estudiantes según edad y sexo.

\begin{tabular}{|c|c|c|c|c|c|c|c|c|c|}
\hline \multirow{2}{*}{ Edad/años } & \multicolumn{3}{|c|}{ Femenino } & \multicolumn{4}{|c|}{ Masculino } & \multicolumn{2}{|c|}{ Total } \\
\hline & $2^{\circ} 3^{\circ}$ & $4^{\circ}$ & $5^{\circ}$ & $2^{\circ}$ & $3^{\circ}$ & $4^{\circ}$ & $5^{\circ}$ & $\mathbf{N}$ & $\%$ \\
\hline 18 & 10 & 0 & 0 & 0 & 0 & 0 & 0 & 1 & 4 \\
\hline 19 & 42 & 0 & 0 & 0 & 1 & 0 & 0 & 7 & 32 \\
\hline 20 & 20 & 2 & 0 & 0 & 0 & 1 & 0 & 5 & 23 \\
\hline 21 & 00 & 1 & 2 & 0 & 0 & 0 & 0 & 3 & 14 \\
\hline 22 & 00 & 1 & 3 & 0 & 0 & 0 & 0 & 4 & 18 \\
\hline $23 a+$ & 00 & 0 & 1 & 0 & 0 & 0 & 1 & 2 & 9 \\
\hline Total & 72 & 4 & 6 & 0 & 1 & 1 & 1 & 22 & 100 \\
\hline
\end{tabular}

Fuente: Estudiantes FAEN/UNJBG

En la Tabla $\mathrm{N}^{\circ} \mathrm{O} 1$ se observa que el $55 \%$ tiene edades entre 19 a 20 años, el $32 \%$ de 21 a 22 años; el sexo predominante es femenino (18) y una minoría es de sexo masculino (3).

De la muestra tomada, se observa que en los primeros años las prácticas pre profesionales se dan por igual en hospitales y comunidad, mientras que en los últimos años hay predominio de práctica comunitaria.

Tabla $\mathbf{N}^{\mathbf{0}} \mathbf{0 2}$. Categorías y subcategorías evaluadas en la Relación de Ayuda como competencia relacional del estudiante, desde la visión Antropológica.

\begin{tabular}{|c|c|c|c|}
\hline CATEGORÍA & \multicolumn{3}{|c|}{ SUB CATEGORÍAS } \\
\hline $\begin{array}{l}\text { Estrategias y } \\
\text { habilidades }\end{array}$ & $\begin{array}{l}\text { Preparación y } \\
\text { conocimientos }\end{array}$ & $\begin{array}{l}\text { Actitudes y } \\
\text { habilidades } \\
\text { practicadas }\end{array}$ & Estilos de relación \\
\hline $\begin{array}{l}\text { Dificultades en la } \\
\text { relación de ayuda }\end{array}$ & $\begin{array}{ll}\text { Tipos } & \text { de } \\
\text { dificultades } & \end{array}$ & $\begin{array}{l}\text { Cómo maneja } \\
\text { las } \\
\text { dificultades }\end{array}$ & $\begin{array}{l}\text { Pensamientos } \\
\text { sentimientos } \\
\text { durante la relación } \\
\text { de ayuda }\end{array}$ \\
\hline $\begin{array}{l}\text { Valor y significado } \\
\text { de la relación de } \\
\text { ayuda }\end{array}$ & $\begin{array}{l}\text { Estudiantes de } \\
\text { pre grado }\end{array}$ & $\begin{array}{l}\text { Institución } \\
\text { (hospitales } \\
\text { comunidad) }\end{array}$ & Conflicto axiológico \\
\hline $\begin{array}{l}\text { Formación en } \\
\text { relación de ayuda }\end{array}$ & $\begin{array}{l}\text { Formación } \\
\text { inicial }\end{array}$ & $\begin{array}{l}\text { Formación } \\
\text { continuada }\end{array}$ & $\begin{array}{l}\text { Resultados de } \\
\text { formación en } \\
\text { Relación de ayuda }\end{array}$ \\
\hline
\end{tabular}

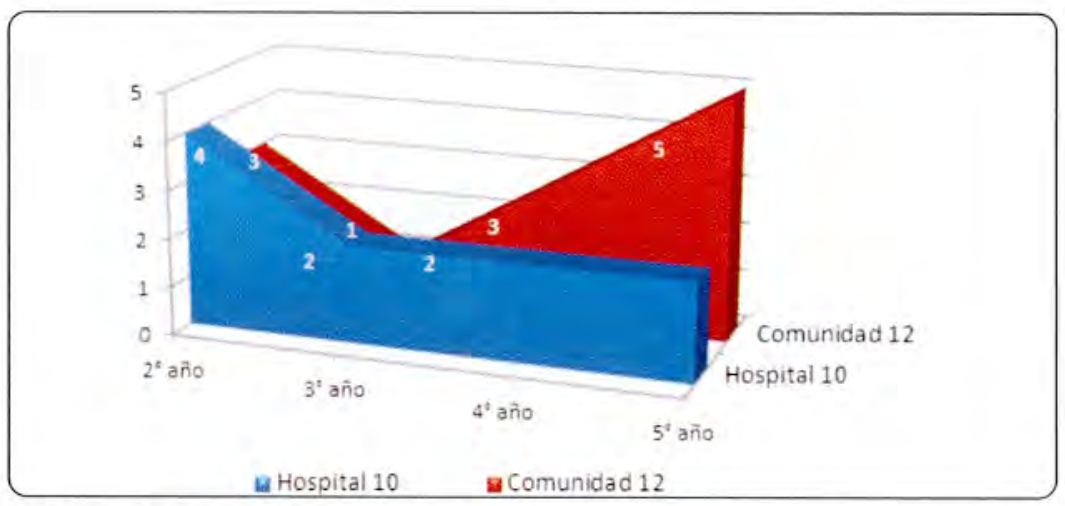

Gráfico $\mathbf{N}^{\circ} \mathbf{o 2}$. Distribución de estudiantes según área de práctica pre profesional. 
Tabla $\mathbf{N}^{\circ}$ 03. Categoría de análisis: Formación adquirida en relación de ayuda con visión antropológica

\begin{tabular}{|c|c|}
\hline $\begin{array}{l}\text { Formación } \\
\text { inicial: } \\
\text { informados } \\
\text { pero no } \\
\text { formados }\end{array}$ & $\begin{array}{l}\text { - Durante la formación inicial, obtuvieron información en algunas asignaturas acerca del } \\
\text { proceso de comunicación y algunas habilidades y actitudes, pero no fueron formados } \\
\text { en cómo desplegarlas adecuadamente en la práctica como parte del cuidado de } \\
\text { enfermeria. } \\
\text { - Disonancia entre la formación inicial y la práctica, van adquiriendo durante su } \\
\text { aprendizaje una identidad como profesionales de enfermería, en donde la Relación de } \\
\text { Ayuda no figura como cuidado importante y necesario para el paciente o familia, en el } \\
\text { marco de sus competencias pre profesionales. }\end{array}$ \\
\hline $\begin{array}{l}\text { Aprendiendo } \\
\text { de la } \\
\text { experiencia } \\
\text { de otros }\end{array}$ & $\begin{array}{l}\text { - Relevancia educativa de estudiantes de años superiores que comparten experiencias } \\
\text { de su formación inicial. } \\
\text { - Formación y transmisión de valores del Jefe de Práctica, considera el estudiante que } \\
\text { fue prácticamente nula y no obtuvieron dicho aprendizaje. } \\
\text { - Los estudiantes que tuvieron la oportunidad de obtener formación y transmisión de } \\
\text { valores por parte del Jefe de Práctica o compañeros de años superiores, se sienten } \\
\text { más seguros y agradecidos para desenvolverse. } \\
\text { - Resulta relevante en el discurso la figura del Jefe de Práctica y compañeros de años } \\
\text { superiores, que conocen el proceso de Relación de Ayuda como potencial educativo, } \\
\text { pues reconocen que ha favorecido la humanización del cuidado brindado. }\end{array}$ \\
\hline $\begin{array}{l}\text { Formación } \\
\text { continuada }\end{array}$ & $\begin{array}{l}\text { - Escasa oferta y demanda en relación directa con el valor secundario que la Relación de } \\
\text { Ayuda tiene dentro de la cultura sanitaria. } \\
\text { - Oferta y demanda que guarda relación directa con la visión biomédica de la } \\
\text { enfermedad, que reduce la Relación de ayuda a la explicación que se le da al paciente } \\
\text { sobre su enfermedad. }\end{array}$ \\
\hline $\begin{array}{l}\text { Propuestas } \\
\text { de mejora }\end{array}$ & $\begin{array}{l}\text { - Más cuidado en la relación directa e indirecta de las asignaturas específicas que guían } \\
\text { al aprendizaje de Relación de Ayuda. } \\
\text { - Mayor oferta en formación continuada o capacitación continua. } \\
\text { - Apoyo institucional educativo y de servicios de salud, que sirvan de base en la } \\
\text { formación v autoavuda para avudar a otros. }\end{array}$ \\
\hline
\end{tabular}

\section{IV.-DISCUSIÓN}

\section{- Formación inicial}

Los estudiantes entrevistados manifiestan que no recibieron formación inicial durante sus años de pre grado, acerca del proceso de relación de ayuda, ya que en el plan de estudios del currículo no existe una asignatura que les enseñe a acompañar y ayudar de manera eficaz a la persona en situación de enfermedad y crisis familiares.

Las asignaturas de Antropología y Sociología, enfatizaron aspectos históricos de la realidad humana y procesos histórico sociales del hombre, en Psicología se enfatiza las corrientes y Teorías más no les capacita para entender o relacionarse con el ser humano, y similares situaciones ocurre con algunas de las pocas asignaturas donde se tratan tópicos generales dentro de habilidades psicosociales pero no específicamente de Relación de Ayuda; debiera de tratarse este aspecto que en la práctica es fundamental para aplicar los cuidados de Enfermería. Los estudiantes de quinto año refieren aun sentir temor y no saber cómo enfrentar situaciones.

\section{- Aprendiendo de la experiencia de otros}

El Docente constituye el modelo a seguir así como otros referentes de años superiores que los estudiantes toman como ejemplo y fuente de consulta, otros manifiestan que no tuvieron la experiencia de aprendizaje en Relación de Ayuda como tal, los estudiantes de años superiores ejercen influencia y dan ejemplo a los de los primeros años, refieren tener experiencias insatisfactorias con los docentes pues la gran mayoría no les tratan ni enseñan con paciencia, especialmente en los primeros años, mientras que los de años superiores (4to. y 5 to.) manifiestan que son años de "tormento", pues tienen mucha presión y los docentes no son cercanos a ellos, consideran que "a nivel docente y jefes de práctica también les falta Relación de ayuda”, en los servicios de salud consideran los estudiantes que la situación es peor, ya que la gran mayoría de profesionales de enfermería y otras afines les ignoran y no les enseñan, obteniendo frases como "estoy ocupada, a tu docente le pagan para que te enseñe..."; lo que les causa en muchos casos impotencia y llanto por la dureza del trato entre el personal. 


\section{- Formación continuada}

El estudiante se preocupa por aprobar lo que le exige el plan de estudios, refieren que pocos se ocupan de su autoeducación que complementen sus estudios de enfermería, toman cursos como idiomas, cómputo, entre algunos. No han visto cursos de relación de ayuda o similares específicos, ya que mayormente se ofertan cursos de aspectos procedimentales y tecnológicos, o eminentemente teóricos. De igual forma comentan que las orientaciones normativas o programas de inducción en las instituciones de salud tienen un enfoque biomédico centrado en el profesional y no en las personas o pacientes, por lo el tema de Relación de ayuda prácticamente es nuevo para ellos.

\section{- Propuestas de mejora}

A partir de las experiencias de competencias relacionales para ayudar al ser humano afectado en su integridad, sin haber tenido el conocimiento específico de la relación de ayuda, los estudiantes por autoeducación se motivaron a compartir aportes, beneficios, resultados e importancia de la misma. Reconocen que la relación de ayuda es un recurso vital para el desarrollo de sus competencias de relación humano a humano, o persona a persona. Sugieren se incluya de manera específica este aspecto en el Plan de estudios, sea en asignatura, unidad ó temario de ellas, así como reorientar los aportes de asignaturas como Antropología, Sociología, Psicología entre otras. Todo cuidado de enfermería es una nueva experiencia con cada ser humano, por tal razón la importancia de saber relacionarse, para saber ayudar.

\section{V.- CONCLUSIONES}

- Las habilidades mayormente desarrolladas en los estudiantes son la comunicación verbal y no verbal, la escucha activa, la compasión, el silencio y la creatividad; entre las actitudes, la empatía mayormente, sin que el conjunto de ellas llegue a ser una Relación de Ayuda propiamente.

- Al no recibir enseñanza específica en Relación de Ayuda, es propio que el estudiante no esté en condiciones de desarrollar su competencia de relacionarse con el ser humano a su cuidado, por ende existe una relación directa entre enseñanza y aplicación de la relación de Ayuda, por lo que el estudiante de pre grado alcanza para este estudio el nivel limitado (mínimo positivo) para esta área.

\section{REFERENCIAS BIBLIOGRÁFICAS}

1. CANOvas TOMAS, Miguel Angel. 1. http://hdl.handle.net/10201/3562. [Citado el: 12 abril a 20 de diciembre de 2010 .]

2. CIBANAL JUAN, L. y colaboradores. VI Edición Programa de de Investigación en Docencia Universitaria. Alicante España: Universidad de Alicante, 2007, Vol. VI.

3. ARRANZ. Relación con el doliente. España: s.n., 2005.

4. BLATUILLE, R. 3ra. Conocerse a sí mismo, un requisito para la Relación de ayuda. Jornada de enfermería en Atención Primaria, Madrid España: s.n., 2002.

5. BERMEJO, JC. s.l. Relación de ayuda a la persona Mayor. : Santander Sal Terrae, 2004.

6. ESTEBAN REDONDO, C. El papel del Antropólogo en el ámbito de la Salud. Murcia España: X Congreso Internacional de Estudiantes de Antropología, 2002.

7. FEITO, L. s.l. La Ética del cuidado como modelo de la Ética. : Metas de Enfermería, 2005.

8. LLEIXÁ, M. s.l. Importancia de las intervenciones psicosociales para la profesión de Enfermería: Metas de Enfermería, 2007.

9. TARRÉS CHAMORRO, S. El cuidado del otro. Gaceta de Antropología, s.l.: Diversidad Cultural y Enfermería transcultural., 2001.

\section{Correspondencia:}

Jenny C. Mendoza Rosado

jenny_concep@hotmail.com

Ciudad Universitario fundo "Los Granados"

Av. Miraglores s/n Tacna - Perú 